\title{
Regional anaesthesia for ambulatory surgery
}

\section{Overview}

Why should we offer regional anaesthesia for ambulatory surgery? There are advantages for the patient. There is no loss of consciousness or "control," for those patients who like to be aware or simply are afraid of general anaesthesia. Recovery time can be decreased and hospital admission rate may be reduced after regional anaesthesia. Regional block can provide postoperative analgesia limited to the operative site, without the side-effects of narcotics. In addition, the hazards and discomforts of general anaesthesia can be avoided or minimized, such as sore throat, airway trauma, muscle pain, and nausea and vomiting.

There are also some disadvantages. Regional anaesthesia requires the active cooperation of patient and surgeon so that the procedure is successful. Regional anaesthesia may take additional time, particularly for epidural and brachial plexus blocks. The induction and maintenance of regional anaesthesia are associated with minor discomfort. Also, there are complications specific to each of the particular blocks and local anaesthetic agents that are used.

\section{Patient choice and preparation}

The most critical element for success with regional anaesthesia is the selection of an appropriate care team, which includes the anesthetist, the surgeon and specific surgical procedure, and the nurse. However, the primary team member is the patient, who must be thoroughly educated about the proposed blocks. Ambulatory regional anaesthesia has been used successfully with retarded adults, with the elderly, and with children to provide postoperative analgesia.'

Patient preparation for regional anaesthesia should be the same as for general anaesthesia, including history, physical examination and minimal laboratory tests; a coagulation profile is not required. If the patient is to receive more than local anaesthesia, monitoring should be the same as for general anaesthesia. Direct $\mathrm{CO}_{2}$ monitoring of ventilation is often possible when the catheter is connected to the nasal prongs or facemask used to provide supplemental oxygen.

\section{Regional anaesthetic techniques}

Success with ambulatory regional techniques is the sum of the right block, the right drug and their proper administra- tion. The techniques to be presented are local infiltration, intravenous regional, peripheral nerve block, brachial plexus block, epidural, and spinal anaesthesia. This discussion will not present technical aspects of the techniques but will, instead, address details of their application to ambulatory surgery.

There are two groups of local anaesthetic agents used for infiltration anaesthesia: short-duration esters, procaine and chloroprocaine (1-2\%) and moderate-duration amides, lidocaine, mepivacaine, and prilocaine (0.5-1\%). For all agents, it is imporant to use dilute solutions, in order to allow use of the larger volumes which are often needed for infiltration. The addition of epinephrine to the local anaesthetic solutions increases both the permissable dose and the duration of action of these drugs. ${ }^{2}$ Longer-acting local anesthetics can be used for the ambulatory surgery patient to provide postoperative analgesia, by infiltration of wound edges or joint cavities. Bupivacaine $0.25 \%$ with epinephrine is commonly employed.

Another limiting factor with local infiltration is the pain associated with the injection itself. There are intrinsic differences in the discomfort caused by different local anaesthetic agents. ${ }^{3}$ Etidocaine and bupivacaine cause the most discomfort, whereas lidocaine and procaine cause the least. The pain caused by these agents can be limited by the addition of sodium bicarbonate, $1 \mathrm{meq} \cdot 10 \mathrm{ml}^{-1}$ lidocaine. ${ }^{4}$ An emulsion of lidocaine $5 \%$ and prilocaine $5 \%$, known as EMLA (eutectic mixture of local anaesthetics), has been developed to anaesthetize the skin surface without injection. ${ }^{5}$ However, the onset of analgesia with this mixture is slow, approximately one hour, which will limit its use especially for the adult ambulatory patient.

Intravenous regional (IVR) anaesthesia is one of the most popular techniques because it is simple and has a high success rate when reasonable care is taken. The keys to success include adequate exsanguination of the extremity and a carefully checked tourniquet. The use of adequate tourniquet inflation pressure, ${ }^{6}$ duration of inflation ${ }^{7}$ as well as cyclic deflation ${ }^{8}$ will limit the release of local anaesthetic into the systemic circulation. A double cuff is suggested, ${ }^{9}$ both to minimize leak and to increase patient comfort: it can be positioned on the upper or lower extremity. The local anaesthetic most commonly used for

From the Department of Anaesthesia, Brigham and Women's Hospital, and Harvard University. 
IVR is lidocaine $3 \mathrm{mg} \cdot \mathrm{kg}^{-1}$. Chloroprocaine is not recommended because it can cause phlebitis. ${ }^{10}$ Bupivacaine also should not be used because of the possibility of serious systemic toxicity, with prolonged myocardial depression." Prilocaine $0.5 \%$ is effective for IVR and the maximum anaesthetic blood concentration is lower than after an equal volume of lidocaine $0.5 \% .^{12}$

Peripheral nerve blocks can be used for extremity procedures. Minor nerve blocks can be performed at the wrist, fingers and ankle. Useful local anaesthetic agents include both the short acting esters and the moderate duration amides. The duration of block, both sensory and motor, can be prolonged by the addition of epinephrine.

The major nerve block most commonly performed for ambulatory anaesthesia is at the brachial plexus. The axillary approach to the brachial plexus is simplest and safest, without risk of pneumothorax; the area anaesthetized is the forearm and hand.' With additional local anaesthetic at the same injection site, anaesthesia can also be provided for tourniquet application. Other approaches to the brachial plexus provide different areas of anaesthesia, but carry increased risk of pneumothorax which will become symptomatic after patient discharge.

The onset of anaesthesia with major nerve blocks can be slow; to facilitate their use in ambulatory surgery patients, these blocks should be placed in a preoperative holding area if possible. The addition of sodium bicarbonate, $1 \mathrm{meq} \cdot 10 \mathrm{ml}^{-1}$ lidocaine or mepivacaine, will also hasten onset. Patients who have had brachial plexus block are often ready for discharge while residual sensory or motor anaesthesia is still present. Patients can be sent home then, with their arm in a sling and with careful written and verbal instructions. ${ }^{13}$

Various minor and major nerve blocks can be combined to provide regional anaesthesia for specialized surgical procedures. These are particularly useful for operations on the scalp, face or oral cavity, as well as retrobulbar and peribulbar ${ }^{14}$ anaesthesia for cataract surgical procedures.

Central neural block can be provided for ambulatory surgery patients, including caudal and lumbar epidural and spinal anesthesia. With epidural block, the onset of anaesthesia can be slow; it can be hastened with the use of ultra short-acting agents such as chloroprocaine, and by starting the block in the preoperative holding area. However, the use of chloroprocaine has been associated with back pain after dissipation of the block. ${ }^{15}$ Epidural anaesthesia can be used for laparoscopic abdominal surgery, but ambulatory patients should be forewarned about the persistence of shoulder pain despite high thoracic anaesthesia. This referred diaphragmatic irritation, and the quality of the block overall, can be improved by adding fentanyl, $50 \mu \mathrm{g}$, to the local anaesthetic solution. Epidural anesthesia can also be used in children, particularly to provide postoperative analgesia.

For spinal anaesthesia, the agent most commonly used is lidocaine, either hyperbaric, $5 \%$, or isobaric, $2 \%$. The longer-acting agents such as bupivacaine or tetracaine should, in general, not be used for ambulatory surgery patients because of the protracted recovery period necessary. The issue of postdural puncture headaches (PDPH) is frequently raised. The incidence of PDPH in ambulatory surgery patients is given as $5.2 \%$ by Mulroy et al. ${ }^{16}$ This can be compared with the incidence of non-spinal headache after non-spinal (general) anaesthesia of $17-26 \%$. Techniques for prevention of PDPH in the typical younger, female ambulatory surgery patient include use of a small gauge, noncutting needle with the bevel parallel to the dural fibres, adequate postoperative hydration and avoidance of Valsalva manoeuvres. ' Confining patients to bed after spinal anaesthesia does not prevent the occurrence of PDPH. Proper postoperative instructions, both oral and written, are important for the ambulatory surgery patient who has had spinal anaesthesia. If PDPH occurs, we suggest conservative therapy consisting of rest, hydration especially with caffeinated beverages, and analgesics. Most PDPH resolve with conservative therapy. ${ }^{17}$ For the few which do not, an epidural blood patch can be performed on an outpatient basis.

\section{Supplemental medications}

Intravenous medications can be given before and continued during ambulatory regional anaesthesia. These supplemental medications serve to relieve fear and anxiety and to improve patient acceptance of the minor discomforts associated with regional anaesthesia. Verbal reassurance and music through headphones are also effective supplements. ${ }^{18}$

One class of drugs used for supplementation of ambulatory regional anesthesia is the sedatives. Diazepam is an older benzodiazepine which is well absorbed orally and can be used for premedication in 5-10 mg doses. Diazepam still enjoys considerable popularity despite several problems, such as venous irritation (Valium ${ }^{\circledR}$ formulation $)^{19}$ and prolonged recovery. ${ }^{20}$ Midazolam is a newer water soluble benzodiazepine which causes minimal pain or phlebitis. Midazolam has a shorter duration of action, with a plasma half-life of $60-90$ minutes and without evidence of enterohepatic recirculation or recurrence of drowsiness. The infusion route is successful for longer procedures. Midazolam is a potent amnestic, not correlated to the degree of drowsiness produced, and caution should be used in giving this drug to patients who have chosen regional anaesthesia to retain awareness of 
the procedure. ${ }^{21}$ Another caution is the synergistic respiratory depression seen when midazolam and opiates are given together. ${ }^{22} \mathrm{~A}$ benzodiazepine antagonist, flumazenil, has undergone trials for reversing sedation after ambulatory regional blocks. The degree of awakening with flumazenil is controllable and side-effects are minimal. ${ }^{23}$

Droperidol is a butyrophenone which produces sedation and a sense of detachment. However, droperidol causes a syndrome of apprehension and anxiety despite apparent drowsiness which has led to cancellation of surgery. Postdischarge anxiety and restlessness have been observed even at low antiemetic doses. ${ }^{24}$

Propofol is the newest agent being used for supplemental sedation during ambulatory regional blocks. Its advantages are rapid recovery, little postoperative emesis and high patient approval. Disadvantages include talkativeness, pain on injection and the high cost. Supplementation with propofol can be given by intermittent bolus but an infusion technique is often more appropriate because of the drug's rapidity of action. Sedative infusions of propofol and midazolam have been compared. ${ }^{25}$ The times to initial awakening were shorter with the shorter-acting propofol. However, the times to recovery - the ability to sit, stand and be ready for discharge - are not different.

Benadryl is a sedative drug with indications for the patient undergoing ambulatory regional anaesthesia. It is effective for neuraxial opiate pruritus, and to reduce Parkinsonian tremors.

Another major class of agents used for supplementation of ambulatory regional anaesthesia is the narcotics. Fentanyl is frequently used. However, it causes respiratory depression, comparable in magnitude and duration to equipotent doses of morphine. ${ }^{26}$ There is also a secondary increase in plasma fentanyl concentrations, associated with recurrence of side-effects. ${ }^{27}$ Sufentanil is a related opiate with somewhat shorter duration of action and more sedative effect.

The opioid alfentanil has a redistribution half-life of $11.6 \mathrm{~min}$ and an elimination half-life of $94 \mathrm{~min}$ compared with 219 min with fentanyl; recovery is therefore shorter. It is also more sedative than the parent compound. Alfentanil is often appropriately given by continuous infusion, with a decrease in the total dose needed and a decrease in recovery time.$^{28}$ However, administration of excessively large doses will lead to accumulation of the drug and prolonged recovery.

In an attempt to provide analgesia with fewer sideeffects, the agonist-antagonist class of opioids has been developed. Nalbuphine and butorphanol both are longlasting, three or more hours. Butorphanol has high patient acceptance $^{29}$ and is a good supplement to ambulatory regional anaesthesia, providing considerable sedation which may reduce or eliminate the need for additional sedative drugs.

The administration of anaesthetic gases and vapours at sub-anaesthetic concentrations can provide analgesia and sedation to supplement regional anaesthesia. The advantages are rapid reversibility and easier maintenance of a constant blood concentration. Currently, nitrous oxide is widely used. It is administered as $30-50 \%$ in oxygen, with maximum psychomotor effects in seven minutes. ${ }^{30}$ Twenty to thirty minutes are needed to complete recovery; the patient should therefore be supervised adequately. Enflurane $0.5 \%$ provides comparable psychomotor impairment and amnesia to $40 \%$ nitrous oxide. ${ }^{31}$

There are side-effects of nitrous oxide inhalation. Nitrous oxide concentrations greater than $30 \%$ may cause excitement. At $50 \%$ nitrous oxide, $21 \%$ of patients experienced minor side-effects which included nausea and vomiting, dizziness, lightheadedness, excitement and numbness; an additional $7.6 \%$ of patients became drowsy or lightly asleep. ${ }^{32}$ However, oversedation can result in loss of airway reflexes. ${ }^{33}$ Inhalation analgesia should be administered only with supervised use.

Regional anaesthesia can cause discomfort while being administered, and it may be advantageous to blunt consciousness temporarily at the time. This technique involves subhypnotic doses of anaesthesia induction agents; therefore, preparation should be the same as for general anaesthesia. Incremental boluses of thiopentone, methohexitone, midazolam or propofol can be used to provide brief sedation; the latter three can also be given by continuous infusion.' The use of ketamine for temporary blunting of consciousness during regional block has been widely reported in the non-anaesthesia literature, but this drug is not recommended due to the high incidence of unpleasant reactions. ${ }^{34}$

In summary, regional anaesthesia can provide intraoperative and postoperative analgesia without postanaesthetic central depression. With the use of appropriate agents and techniques, and supplemental sedation as needed, the disadvantages of regional block can be minimized and potential complications may be avoided. Regional anaesthesia for the ambulatory patient can truly satisfy patient, surgeon and anaesthetist.

\section{References}

1 Philip BK, Covino BG. Local and regional anesthesia. In: Wetchler BV (Ed.). Anesthesia for Ambulatory Surgery. Philadelphia: JB Lippincott, 1991; 309.

2 Philip BK. Local anesthesia and sedation techniques. In: White PF (Ed.). Outpatient Anesthesia. New York: Churchill Livingstone, 1990; 263. 
3 Watcha $M$, White $P F$. Postoperative nausea and vomiting. Its etiology, treatment and prevention. Anesthesiology 1992; 77: 162-84.

4 Carmichael J, Cantwell BMJ, Edwards CM, et al. A pharmacokinetic study of granisetron (BRL 43694A), a selective 5- $\mathrm{HT}_{3}$ receptor antagonist: correlation with anti-emetic response. Cancer Chemother Pharmacol 1989; 24: 45-9.

5 Furue $H$, Oota $K$, Taguchi $T$, Niitani $H$. Clinical evaluation of granisetron against nausea and vomiting induced by anticancer drugs. (I) Optimal dose-finding study. Journal of Clinical and Therapeutic Medicine 1990; 6: 49-61 (Japanese).

6 Falkson G, van $Z y l$ AJ. A phase I study of a new $5 \mathrm{HT}_{3^{-}}$ receptor antagonist, BRL43694A, an agent for the prevention of chemotherapy-induced nausea and vomiting. Cancer Chemother Pharmacol 1989; 24: 193-6. 


\section{Anesthésie régionale en chirurgie ambulatoire}

Pourquoi devrions-nous offrir l'anesthésie régionale en chirurgie ambulatoire ? Il y a plusieurs avantages pour le patient. Il n'y a pas de perte de conscience ou de « contrôle » pour ces patients qui préfèrent demeurer éveillés ou simplement ont peur de l'anesthésie générale. Le temps de récupération peut être diminué et le taux d'admission à l'hôpital peut être réduit après l'anesthésie régionale. Un bloc régional procure une anesthésie postopératoire limitée au site opératoire, sans les effets secondaires des narcotiques. De plus, l'inconfort et les inconvénients de l'anesthésie générale tels la gorge irritée, le traumatisme des voies aériennes, les douleurs musculaires et les nausées et vomissements sont évités ou diminués.

Il y a aussi quelques désavantages. L'anesthésie régionale requiert la coopération active du patient et du chirurgien pour que l'intervention soit réussie. L'anesthésie régionale peut prendre plus de temps, surtout lorsqu'on pense aux blocs épiduraux et du plexus brachial. L'induction et l'entretien de l'anesthésie régionale s'accompagnent d'un certain degré d'inconfort. De plus, il y a des complications spécifiques à chaque bloc et chaque anesthésique local utilisé.

\section{Choix du patient et préparation}

L'élément le plus important pour s'assurer du succès de l'anesthésie régionale est la sélection d'une équipe appropriée comprenant l'anesthésiste, le chirurgien et son intervention spécifique, et l'infirmière. Cependant, le membre principal de l'équipe est le patient, et il doit bien connaître les caractéristiques des blocs proposés. L'anesthésie régionale ambulatoire a été utilisée avec succès chez des retardés mentaux, chez les gens âgés et les enfants pour leur procurer une analgésie postopératoire. ${ }^{\text {' }}$

La préparation du patient pour l'anesthésie régionale devrait être identique à celle prévue pour l'anesthésie générale, et inclure l'histoire clinique, l'examen physique et un nombre minimal d'examens de laboratoire ; le profil de coagulation n'est pas nécessaire. En dehors d'une anesthésie locale, le monitoring du patient devrait être le même que pour l'anesthésie générale. La mesure directe du $\mathrm{CO}_{2}$ est souvent possible par un cathéter branché sur les canules nasales ou le masque facial utilisé pour donner de l'oxygène supplémentaire.

Techniques d'anesthésie régionale

La réussite des techniques d'anesthésie régionale ambu- latoire est la somme combinée du bon choix du bloc, d'agent anesthésique et son administration appropriée. Les techniques présentées sont l'infiltration locale, l'anesthésie intraveineuse, le bloc de nerf périphérique, le bloc du plexus brachial, l'anesthésie épidurale et sous-arachnoïdienne. Cette présentation n'insistera pas sur les techniques mais plutôt sur les détails d'application à la chirurgie ambulatoire.

Il y a deux groupes d'agents anesthésiques locaux en usage pour l'anesthésie d'infiltration: les esters de courte durée, procaïne et chloroprocaïne (1-2\%), et les amides de durée moyenne, lidocaïne, mépivacaïne et prilocaïne $(0,5-1 \%)$. Pour tous ces agents il est important d'utiliser une solution diluée, ce qui permet d'utiliser les volumes importants souvent nécessaires pour l'infiltration. L'addition d'adrénaline à la solution anesthésique locale augmente autant la dose permise que la durée d'action de ces substances. ${ }^{2}$ Les anesthésiques locaux à longue durée d'action peuvent être utilisés pour la chirurgie ambulatoire pour donner une analgésie postopératoire, en infiltrant les parois d'incision ou les cavités articulaires. La bupivacaïne $0,25 \%$ avec adrénaline est souvent employée.

Une des limites de l'infiltration locale est la douleur associée avec l'injection elle-même. Il y a des différences intrinsèques dans l'inconfort causé par les différents agents anesthésiques locaux. ${ }^{3}$ L'étidocaïne et la bupivacaïne causent le plus d'inconfort, alors que la lidocaine et la procaïne en causent moins. La douleur amenée par ces agents peut être limitée par l'addition de bicarbonate de sodium, $1 \mathrm{meq} \cdot 10 \mathrm{ml}^{-1}$ de lidocaïne. ${ }^{4}$ Une émulsion de lidocaine $5 \%$ et de prilocaine $5 \%$ connue comme l'EMLA (mélange eutectique d'anesthésiques locaux), a été mise au point pour anesthésier la peau en surface sans injection. ${ }^{5}$ Cependant, le début de l'analgésie avec ce mélange est lent, d'environ une heure, et limite son utilisation, en particulier chez les adultes soumis à une chirurgie ambulatoire.

L'anesthésie régionale intraveineuse est une des techniques les plus populaires parce qu'elle est simple et a un taux de réussite élevé lorsque la technique est soigneuse. Les clefs du succès comprennent une exsanguination adéquate de l'extrémité à anesthésier et une vérification attentive du tourniquet. L'utilisation d'une pression d'insufflation adéquate du tourniquet, ${ }^{6}$ la durée de l'insufflation ${ }^{7}$ ainsi que la déflation périodique ${ }^{8}$ vont diminuer la libération d'anesthésiques locaux dans la circulation 
systémique. On suggère un double tourniquet ${ }^{9}$ autant pour diminuer les fuites que pour améliorer le confort du patient; il peut être placé sur l'extrémité supérieure ou inférieure. L'anesthésique local le plus couramment utilisé pour l'anesthésie régionale intraveineuse est la lidocaïne à raison de $3 \mathrm{mg} \cdot \mathrm{kg}^{-1}$. La chloroprocaïne n'est pas recommandée parce qu'elle peut causer des phlébites. ${ }^{10} \mathrm{La}$ bupivacaïne ne devrait pas être non plus utilisée à cause de la possibilité de toxicité systémique sévère, avec dépression prolongée du myocarde. ${ }^{11}$ La prilocaïne $0,5 \%$ est efficace pour l'anesthésie régionale intraveineuse et le niveau plasmatique maximal est inférieur à celui obtenu après un volume équivalent de lidocaïne $0,5 \% .^{12}$

On peut utiliser un bloc des nerfs périphériques pour la chirurgie des membres. Des blocs de nerfs mineurs peuvent être faits au poignet, au doigt et à la cheville. Les anesthésiques locaux utiles dans cette situation comprennent autant les esters à courte durée d'action que les amides à durée d'action moyenne. La durée du bloc sensitif et moteur peut être prolongée par l'addition d'adrénaline.

Le bloc de nerfs majeurs le plus couramment utilisé en anesthésie ambulatoire se fait au niveau du plexus brachial. L'approche axillaire est la plus simple et la plus sûre, sans risque de pneumothorax ; la région anesthésiée est l'avant-bras et la main. ${ }^{1}$ Un volume additionnel d'anesthésique local au même site d'injection permet l'utilisation d'un tourniquet. D'autres approches du plexus brachial donnent des zones différentes d'anesthésie, mais comportent un risque de pneumothorax qui deviendra symptomatique après le départ du patient.

Le début de l'anesthésie avec les blocs de nerfs majeurs peut être lent; pour faciliter leur utilisation en chirurgie ambulatoire, ces blocs devraient être exécutés si possible dans une salle d'induction. L'addition de bicarbonate de sodium ( $1 \mathrm{meq} \cdot 10 \mathrm{ml}^{-1}$ de lidocaïne ou de mépivacaïne) va aussi accélérer le début d'action. Les patients qui ont eu un bloc du plexus brachial sont souvent prêts à être congédiés même s'il y a une anesthésie résiduelle sensitive ou motrice. Les patients peuvent être renvoyés à la maison, avec leur bras en écharpe et des instructions écrites et verbales précises. ${ }^{13}$

Plusieurs blocs de nerfs majeurs et mineurs peuvent être combinés pour procurer de l'anesthésie régionale lors d'interventions particulières. Ces combinaisons sont particulièrement utiles pour les interventions sur le scalp, la face ou la cavité orale, ainsi que lors d'anesthésie rétrobulbaire et péribulbaire pour chinurgie de cataracte. ${ }^{14}$

Les blocs centraux peuvent être utiles en chirurgie ambulatoire: ils incluent l'anesthésie épidurale lombaire et caudale et l'anesthésie sous-arachnoïdienne. Avec le bloc épidural le début de l'anesthésie peut être lent ; il sera accéléré en utilisant des agents de durée d'action ultra- courte comme la chloroprocaïne, et en exécutant le bloc dans une salle d'induction. Cependant l'utilisation de la chloroprocaïne a été associé avec des douleurs dorsales une fois le bloc disparu. ${ }^{15} \mathrm{~L}$ 'anesthésie épidurale peut être employée pour la chirurgie abdominale laparoscopique, mais les patients devront être avisés de la possibilité de douleur à l'épaule malgré une anesthésie s'étendant jusqu' au milieu du thorax. Cette irritation diaphragmatique référée, et la qualité totale du bloc, peuvent être améliorées en ajoutant $50 \mu \mathrm{g}$ de fentanyl à la solution anesthésique locale. L'anesthésie épidurale pourra aussi être utilisée chez les enfants, surtout pour leur donner une analgésie postopératoire.

En ce qui concerne l'anesthésie sous-arachnoïdienne, l'agent le plus fréquemment utilisé sera la lidocaïne, soit hyperbare, $5 \%$ ou isobare, $2 \%$. Les agents de longue durée d'action telle la bupivacaïne ou la tétracaïne devraient, en général, être évités en chirurgie ambulatoire à cause de la longue période de récupération nécessaire. Le problème des céphalées post-rachidiennes est souvent soulevé. L'incidence en chirurgie ambulatoire est de l'ordre de $5,2 \%$ selon Mulroy et al. ${ }^{16}$ Ceci est comparable à l'incidence de céphalée non rachidienne après une anesthésie générale qui est de l'ordre de 17 à $26 \%$. Les techniques de prévention de la céphalée post-rachidienne chez la jeune femme soumise à une chirurgie ambulatoire comprennent l'utilisation d'une aiguille de petit calibre, non tranchante, le biseau utilisé parallèlement aux fibres de la dure-mère, une hydratation postopératoire adéquate et l'abstention des manoeuvres de Valsalva. ${ }^{\text {L L'alitement }}$ des patients après une anesthésie sous-arachnoïdienne ne prévient pas l'apparition des céphalées post-rachidiennes. Des. instructions appropriées en postopératoire, autant écrites que verbales, sont importantes chez les patients soumis à la chirurgie ambulatoire sous anesthésie sousarachnoïdienne. Si les céphalées se produisent, nous suggérons une thérapie conservatrice comprenant du repos, une hydratation surtout avec des breuvages caféinés, et des analgésiques. La plupart des céphalées post-rachidiennes disparaissent avec un traitement conservateur. ${ }^{17}$ Pour celles qui ne disparaissent pas, on peut faire un colmatage par caillot sur une base externe.

\section{Médication supplémentaire}

Une médication intraveineuse pourra être donnée avant et pendant l'anesthésie régionale ambulatoire. Ces suppléments servent à diminuer la peur et l'anxiété et à améliorer chez le patient la tolérance à l'inconfort modéré associé à l'anesthésie régionale. La réassurance verbale et la musique diffusée à travers des écouteurs sont aussi des suppléments efficaces. ${ }^{18}$

Les sédatifs constituent une des classes de substances utilisées pour supplémenter l'anesthésie régionale ambu- 
latoire. Le diazépam est une vieille benzodiazépine qui est bien absorbée par voie orale et peut être utilisée comme prémédication à des doses de 5 à $10 \mathrm{mg}$. Le diazépam est toujours populaire malgré certains problèmes tels l'irritation veineuse ${ }^{19}$ et une récupération plus lente. ${ }^{20}$ Le midazolam est une benzodiazépine hydrosoluble plus récente qui cause beaucoup moins de douleurs ou de phlébites. Cette substance a une durée d'action plus courte, avec une demie-vie plasmatique de 60 à 90 minutes et sans évidence de recirculation entérohépatique ou de réapparition de somnolence. L'administration par infusion est utile pour les chirurgies prolongées. Le midazolam a des propriétés amnésiantes puissantes, sans corrélation avec le degré de somnolence produit, et on devrait être prudent lorsqu'on utilise cette substance chez des patients qui ont choisi l'anesthésie régionale pour être " présents " à l'intervention. ${ }^{21}$ Il faut surveiller aussi la dépression respiratoire possible amenée par la synergie produite lors de l'utilisation de midazolam et d'opiacés. ${ }^{22}$ Un antagoniste des benzodiazépines, le flumazenil, a subi des essais pour vérifier sa capacité de renverser la sédation après l'anesthésie régionale ambulatoire. Le degré de réveil avec le flumazenil est contrôlable et ses effets secondaires sont minimes. ${ }^{23}$

Le dropéridol est une butyrophénone qui produit une sédation et une sensation de détachement. Cependant, il cause un syndrome d'appréhension et d'anxiété malgré l'apparence de sédation, et ceci a déjà conduit à l'annulation de la chirurgie. L'anxiété et l'agitation après le congé ont pu être observés même à de faibles doses anti-émétiques. ${ }^{24}$

Le propofol est l'agent le plus nouveau utilisé pour supplémenter les blocs régionaux sur une base ambulatoire. Ses avantages sont une récupération rapide, peu de nausées postopératoires et un très haut taux d'acceptation par les patients. Ses désavantages comprennent la logorrhée, la douleur à l'injection et un coût élevé. La supplémentation au propofol peut être donnée par bolus intraveineux mais l'administration par infusion est souvent plus appropriée à cause de la rapidité d'action de la substance. Des infusions sédatives de propofol et midazolam ont été comparées. ${ }^{25}$ Le réveil initial est plus rapide avec le propofol. Cependant, le temps nécessaire pour la récupération - la capacité de s'asseoir, de se tenir debout et d'être prêt à partir - n'est pas différent.

Le diphenhydramine est une substance sédative indiquée chez les patients qui subissent une anesthésie régionale ambulatoire. Cette substance est efficace pour le prurit dû aux opiacés, et pour réduire les tremblements parkinsoniens.

Une autre classe importante d'agents utilisés pour supplémenter une anesthésie régionale ambulatoire est celle des opiacés. Le fentanyl est souvent utilisé. Ce- pendant, il amène une dépression respiratoire, comparable en importance et en durée à des doses équivalentes de morphine. ${ }^{26} \mathrm{Il}$ y a aussi une augmentation secondaire de la concentration plasmatique du fentanyl, qui s'accompagne de réapparition d'effets secondaires. ${ }^{27}$ Le sufentanil est un opiacé apparenté avec une durée d'action un peu plus courte et plus d'effets sédatifs.

L'alfentanil a une demie-vie de redistribution de 11,6 minutes et une demie-vie d'élimination de 94 minutes, comparée au 219 minutes du fentanyl ; la récupération est dès lors plus rapide. L'alfentanil est aussi plus sédatif que le fentanyl. Il est souvent administré avec profit par infusion continue, ce qui amène une diminution de la dose totale nécessaire et un raccourcissement de la période de récupération. ${ }^{28}$ Cependant, l'administration de doses trop importantes amènera une accumulation de la substance et une récupération prolongée.

La classe des opiacés agonistes-antagonistes a été développée dans le but de produire une analgésie avec moins d'effets secondaires. La nalbuphine et le butorphanol sont deux substances de longue durée d'action, trois heures et plus. Le butorphanol est très bien accepté par les patients ${ }^{29}$ et constitue un supplément intéressant pour l'anesthésie régionale ambulatoire, parce qu'il amène une sédation importante qui peut réduire ou éliminer le besoin d'autres sédatifs.

L'administration de gaz et de vapeurs anesthésiques à des concentrations sub-anesthésiques peut donner de l'analgésie et de la sédation en supplément à l'anesthésie régionale. Les avantages sont une réversibilité rapide et un maintien plus facile d'une concentration constante dans le sang. Actuellement, le protoxyde d'azote est largement utilisé. Il est administré en concentration de 30 à $50 \%$ dans l'oxygène, avec des effets psychomoteurs maximaux atteints en 7 à 8 minutes. ${ }^{30}$ Vingt à 30 minutes sont nécessaires pour une récupération complète; les patients devraient alors être surveillés adéquatement. L'enflurane à $0,5 \%$ procure une amnésie et une atteinte psychomotrice comparable à celle d'une concentration de $40 \%$ de protoxyde d'azote. ${ }^{31}$

Il y a des effets secondaires à l'inhalation de protoxyde d'azote. Des concentrations plus importantes que $30 \%$ amènent de l'excitation. À un niveau de $50 \%$ de $\mathrm{N}_{2} \mathrm{O}, 21 \%$ des patients présentent des effets secondaires mineurs comprenant nausées et vomissements, étourdissement, excitation, engourdissement, et un pourcentage additionnel de 7,6\% des patients deviennent stuporeux ou ont un sommeil léger. ${ }^{32}$ Cependant, une sédation excessive peut amener une perte des réflexes des voies aériennes. ${ }^{33}$ L'analgésie par inhalation devrait être administrée sous supervision seulement.

L'anesthésie régionale peut amener de l'inconfort pendant son administration, et il peut être avantageux de 
modifier l'état de conscience temporairement à ce moment-là. Cette technique implique l'utilisation de doses sous-hypnotiques d'agents d'induction de l'anesthésie ; dès lors la préparation devrait être la même que pour l'anesthésie générale. Des doses progressives de thiopental, de méthohexital, de midazolam ou de propofol pourront être employées pour procurer une sédation de courte durée; le propofol peut être aussi administré en infusion continue. ${ }^{1}$ L'utilisation de la kétamine pour obtenir un émoussement temporaire de l'état de conscience pendant l'anesthésie régionale a été fréquemment mentionnée dans la littérature non-anesthésique, mais cette substance n'est pas recommandée à cause de l'incidence élevée de réactions déplaisantes. ${ }^{34}$

En résumé, l'anesthésie régionale peut procurer une analgésie intraopératoire et postopératoire sans dépression centrale post-anesthésique. En utilisant des agents et des techniques appropriées, et un supplément de sédation lorsque jugé nécessaire, les désavantages de l'anesthésie régionale peuvent être réduits et les complications potentielles évitées. L'anesthésie régionale chez le patient ambulatoire peut véritablement satisfaire le patient, le chirurgien et l'anesthésiste.

\section{Références}

(Voir page R5) 